\title{
DESEMPENHO MOTOR EM IDOSOS PARTICIPANTES DE GRUPOS DE CONVIVÊNCIA
}

\author{
Camila Porto Nascimento*, Lara Sodré Lago*, Rebeca Fernanda Ferraz de Almeida*, Mayra Ferraz \\ Santos Gusmão*, Stênio Fernando Pimentel Duarte**, Luciana Araújo Dos Reis*** \\ Autor correspondente: Luciana Araújo dos Reis. E-mail: lucianauesb@yahoo.com.br. \\ * Graduanda do Curso de Fisioterapia da Faculdade Independente do Nordeste \\ ** Biólogo. Doutor em Fisiopatologia Clínica e Experimental pela Universidade do Estado do Rio de Janeiro, professor da \\ Faculdade Independente do Nordeste \\ *** Fisioterapeuta. Doutora em Ciências da Saúde pela Universidade Federal do Rio Grande do Norte, professora da \\ Faculdade Independente do Nordeste
}

\section{Resumo}

Objetivo: Avaliar o desempenho motor de idosos participantes de grupos de convivência. Método: Pesquisa analítica com delineamento transversal e abordagem quantitativa realizada em um grupo de convivência de idosos, no município de Vitória da Conquista/BA. Resultados: Verificou-se no presente estudo que houve uma maior frequência do sexo feminino ( $92,7 \%)$, idade $\geq 68$ anos $(53,7 \%)$ e viúvos $(37,8 \%)$. Quanto às condições de saúde constatou-se uma maior distribuição de idosos que apresentavam doenças $(80,5 \%)$, sentiam dor $(63,4 \%)$, consideravam-se com boa saúde $(35,4 \%)$. Na avaliação do desempenho motor observou-se uma maior predominância de idosos com limitação na atividade agachar e pegar um lápis no chão $(85,4 \%)$, sem limitação na atividade sentar e levantar $(92,7 \%)$, sem limitação no teste de caminhada $(96,3 \%)$, sem limitação na força de preensão manual $(79,3 \%)$ e sem limitação na atividade de equilíbrio $(81,7 \%)$. Conclusão: Constatou-se que os idosos avaliados apresentaram limitação no desempenho motor apenas na atividade de atividade agachar e pegar um lápis no chão.

Palauras-chave: Envelhecimento; Condições de saúde e desempenho motor.

\section{PERFORMANCE MOTOR IN ELDERLY OF PARTICIPANTS GROUPS OF COEXISTENCE}

\begin{abstract}
Objective: To evaluate the motor performance of elderly participants in social groups. Method: analytical research with cross-sectional design and quantitative approach carried out in a group of elderly living in the municipality of Vitoria da Conquista / BA. Results: It was found in this study that there was a higher
\end{abstract}


frequency of females $(92.7 \%)$, age $\geq 68$ years $(53.7 \%)$ and widowed $(37.8 \%)$. As the health conditions we have seen a greater distribution of seniors who had diseases (80.5\%) felt pain $(63.4 \%)$ considered themselves to be in good health (35.4\%). In the evaluation of engine performance there was a predominance of older people with limited activity in squat and pick up a pencil on the floor (85.4\%) without limitation in the activity sitting and standing $(92.7 \%)$, with no limitation on testing walk (96.3\%), with no limitation in handgrip strength (79.3\%) and without limitation in the balance of activity (81.7\%). Conclusion: It was found that the evaluated subjects presented limitation in motor performance only in the squat activity activity and grab a pencil on the floor.

Keywords: Aging; Health and motor performance conditions.

\section{INTRODUÇÃO}

O aumento da expectativa de vida é hoje um fenômeno universal, o que tornar predominante as doenças crônico-degenerativas e suas complicações, como a perda da autonomia e a independência funcional.(1) Segundo o Instituto Brasileiro de Geografia e Estatística, (2) a população de idosos representa um contingente de quase 15 milhões de pessoas com 60 anos ou mais de idade $(8,6 \%$ da população brasileira). Nos próximos 20 anos, a população idosa do Brasil poderá ultrapassar os 30 milhões de pessoas e deverá representar quase $13 \%$ da população ao final deste período.

O envelhecimento torna o corpo mais vulnerável causando dependência em atividades de vida diária (AVD); doença crônica incapacitante; comprometimento dos mecanismos de homeostase; diminuição da força muscular, mobilidade e equilíbrio, conferindo risco à qualidade de vida e altos custos com a saúde. ${ }^{(3)}$

Durante o processo de envelhecimento ocorre a redução da força muscular que leva a diminuição das fibras musculares e, consequentemente, da massa muscular durante o final da meia idade e dos anos posteriores da idade adulta. A redução das fibras musculares gera comprometimento do desempenho neuromuscular, evidenciado pela fraqueza muscular, pela lentidão dos movimentos, pela perda da força muscular e pela fadiga muscular precoce, constituindo um aspecto marcante do envelhecimento, e trazendo como consequência limitações funcionais para caminhar, levantar-se, e manter o equilíbrio postural, assim como, quedas iminentes. Estas limitações levam a dificuldades na execução das atividades da vida diária (AVD), à dependência funcional e à incapacidade. ${ }^{(4)}$

Essas perdas aleatórias conforme um indivíduo envelhece gera uma deterioração global de desempenho em diversas tarefas, dentre elas, as sensório-motoras. Diante dos diversos indicadores a serem considerados na avaliação das condições de saúde de populações idosas, o desempenho motor ocupa lugar peculiar, pois expressa as habilidades físicas e mentais necessárias à vida independente e autônoma, mesmo quando considerada a presença de enfermidades. ${ }^{(5)}$

Estratégias de atendimento por meio de centros de convivência, que consistem em atividades que visam o fortalecimento de atividades associativas, produtivas e de promoção da sociabilidade contribuem para autonomia e a prevenção do isolamento social. Essa alternativa busca inserir o idoso em diferentes espaços sociais, oferecendo serviços que atendam suas necessidades especificas, visando uma melhor qualidade de vida. ${ }^{(6)}$

Pesquisas avaliaram o desempenho motor de idosos que participam de grupos de convivência e verificou-se que estes são mais ativos fisicamente. (6) Quanto mais atuantes e integrados em seu meio 
social, menos ônus trarão para a família e para os serviços de saúde.

O desempenho motor do idoso está ligado a modificações fisiológicas, anatômicas e psicológicas do processo de envelhecimento e na medida em que este envelhece torna-se susceptível a distúrbios motores, dificultando sua locomoção e por consequente a sua independência e autonomia. $\mathrm{O}$ presente estudo teve como objetivo avaliar o desempenho motor de idosos participantes de grupos de convivência.

\section{MATERIAL E MÉTODOS}

Trata-se de uma pesquisa do tipo analítica com delineamento transversal e abordagem quantitativa. O local de estudo foi um grupo de convivência de idosos, no município de Vitória da Conquista/BA. A amostra do estudo foi composta por 164 idosos que frequentaram o centro de convivência no período da coleta. Sendo indivíduos com 60 anos ou mais de ambos os sexos, apresentando condições mentais para responder a aplicação do instrumento de pesquisa (pontuação acima de 24 pontos no MEEM) e que aceitassem participar voluntariamente do estudo. As condições Mentais foram avaliadas pelo Mini Exame- Estado Mental (MEEM), na versão proposta de Pfeiffer. ${ }^{(7)}$

$\mathrm{O}$ instrumento de pesquisa foi constituído de dados sóciodemográficas: sexo (masculino ou feminino), idade (coletada em anos completos), estado civil (sem companheiro, união estável, solteiro, separado/desquitado/divorciado, viúvo, não sabe/ não respondeu), profissão e renda; Condições de saúde: presença e tipos de problemas de saúde; presença e tipos de sequelas; realização de tratamento; uso de medicamentos; auto percepção do estado de saúde; Avaliação do desempenho motor: Realizada por meio dos testes de Flexibilidade/mobilidade, Teste de Sentar e Levantar (TSL), Teste de caminhar 2,44m, Equilíbrio e Força de Preensão manual.

- Flexibilidade/mobilidade: O teste "agachar e pegar o lápis no chão" proposto para observar a flexibilidade/mobilidade dos músculos posteriores da perna e para verificar a capacidade de abaixar e levantar dos idosos. O indivíduo ficava em posição ereta com os pés juntos, agachava-se e apanhava o lápis a 30 centímetros à frente da ponta dos seus pés. Ficava novamente em pé no tempo de 30 segundos. Foi observado se o indivíduo conseguiu cumprir o teste ou não, se sim, em quanto tempo conseguiu realizá-lo. ${ }^{(8)}$

- Teste de Sentar e Levantar (TSL): Teste utilizado como medida de força de membros inferiores em indivíduos idosos. O indivíduo iniciava o teste em sedestação, com a coluna ereta e os braços cruzados sobre o peito, pés afastados à largura dos ombros e totalmente apoiados no solo e um dos pés deve estar ligeiramente avançado em relação ao outro para ajudar a manter o equilíbrio. $O$ indivíduo devia se levantar cinco vezes, o mais rápido possível, sem fazer nenhuma pausa. O teste é considerado concluído com êxito quando realizado em tempo igual ou inferior a 60 segundos. ${ }^{(8)}$

- Teste de caminhar 2,44m: Utilizado para avaliar a mobilidade física, velocidade, agilidade e equilíbrio dinâmico. $\mathrm{O}$ indivíduo caminhava no trajeto marcado no chão $(2,44 \mathrm{~m})$, em sua velocidade normal, como se estivesse caminhando na rua, sendo verificado se o mesmo conseguia ou não realizar o teste e quantos segundos era gasto pela primeira e segunda vez.

- Equilíbrio: Utilizado para avaliar o equilíbrio estático. Inicialmente o individuo permanecia em pé, por 10 segundos, mantendo os pés juntos e olhos abertos. Depois deveria permanecer em pé, por 1osegundos, colocando o calcanhar de um pé na frente do outro, mantendo os olhos abertos. Posteriormente o individuo deveria permanecer em pé, apoiando-se na outra perna, 10 segundos, sem utilizar qualquer tipo de apoio. Finalmente o individuo deveria permanecer em pé, apoiando- se na outra perna por $10 \mathrm{se}-$ gundos sem utilizar qualquer tipo de apoio. $O$ avaliado era classificado como incapaz se não conseguisse realizar nenhuma tarefa, fraco, se não fosse capaz de realizar somente a tarefa 1 , médio, se fosse capaz de realizar somente a tarefa 1 e 2 e bom se fosse capaz de realizar somente a tarefa 1 e 2 mais a 3 e/ou a $4 .{ }^{(8)}$ 
- A força de preensão manual: foi avaliada através de um dinamômetro manual hidráulico SAEHAN modelo $\mathrm{SH}_{500}$, ajustou-se o aparelho para cada indivíduo de acordo com o tamanho das mãos, realizando três medidas no braço esquerdo e direito dos participantes, de forma alternada por segmento. Em cada uma das medidas o examinado estava sentado com o ombro em posição neutra, cotovelo a $90^{\circ}$, antebraço na posição neutra e o punho entre $\circ$ a $30^{\circ}$ de extensão e $\circ$ a $15^{\circ}$ de desvio ulnar e o indivíduo fez um aperto no dinamômetro com a força das mãos e no final o registro da força foi estabelecida em quilogramas/força $[\mathrm{kg} / \mathrm{f}]$. Foi realizada três tentativas com um período de recuperação de um minuto entre elas e a média das três será o valor da FPM. ${ }^{12}$ Os idosos submetidos a alguma cirurgia no braço ou na mão nos três meses anteriores à coleta dos dados foram excluídos do teste.

Os dados foram analisados por meio do Programa Estatístico Statistical Package for the Social Sciences for Windows (SPSS, versão 20.0), sendo realizada análise estatística descritiva. O proje- to foi aprovado pelo Comitê de Ética em Pesquisa com Seres Humanos da Universidade Estadual do Sudoeste da Bahia e aprovada com o $n^{\circ}$ de protocolo 16450513.6 .0000 .0055 obedecendo às normas de éticas exigidas pela Resolução $n^{\circ} 466,2012$ (Conselho Nacional de Saúde), incluindo a obtenção do Termo de Consentimento Livre e Esclarecido por escrito de cada participante.

\section{RESULTADOS}

Verificou-se no presente estudo uma maior distribuição de idosos do sexo feminino (92,7\%), na faixa etária maior igual a 68 anos $(53,7 \%)$, com renda menor igual a 1 (um) salário mínimo $(74,4 \%)$, estado civil de viuvez $(37,8 \%)$ e profissão referente a dona de casa $(74,4 \%)$.

Tabela 1 - Distribuição dos idosos participante de grupos de convivência segundo dados sociodemográficas. Vitória da Conquista/BA, 2015

\begin{tabular}{lcc}
\hline & $\mathbf{N}$ & $\%$ \\
\hline Sexo & 152 & 92,7 \\
$\quad$ Feminino & 12 & 7,3 \\
$\quad$ Masculino & & \\
Faixa Etária & 88 & 53,7 \\
$\quad \geq 68$ anos & 76 & 46,3 \\
$\quad<68$ anos & & \\
Renda & 122 & 74,4 \\
$\quad 1$ Salário Mínimo & 42 & 25,6 \\
$\quad>1$ Salário Mínimo & \multicolumn{2}{c}{} \\
Estado Civil & 58 & 35,4 \\
$\quad$ União estável & 24 & 14,6 \\
$\quad$ Solteiro (a) & 62 & 37,8 \\
$\quad$ Viúvo (a) & 20 & 12,2 \\
$\quad$ Separado (a)/desquitado (a)/divorciado (a) & 164 & 100,0 \\
\hline Total & & \\
\hline
\end{tabular}

$\mathrm{Na}$ avaliação das condições de saúde verificou-se que houve uma maior frequência de idosos com presença de doenças $(80,5 \%)$, com presen- ça de dor $(63,4 \%)$ e que percebem a sua saúde comparando com pessoas de sua idade como boa $(35,4 \%)$. 
Tabela 2 - Distribuição dos idosos participantes de grupo de convivência segundo as condições de saúde. Vitória da Conquista/BA, 2015

\begin{tabular}{lcc}
\hline & $\mathbf{N}$ & $\%$ \\
\hline Presença de Doenças & 132 & 80,5 \\
Sim & 32 & 19,5 \\
Não & & \\
Presença de Dor & 104 & 63,4 \\
Sim & 60 & 36,6 \\
Não & & 17,1 \\
Auto - Percepção de saúde & 28 & 22 \\
Excelente & 36 & 35,4 \\
Muito Boa & 58 & 18,3 \\
Boa & 30 & 7,3 \\
Razoável & 12 & 100,0 \\
Péssima & 164 & \\
\hline Total & & \\
\hline
\end{tabular}

$\mathrm{Na}$ avaliação do desempenho motor observou-se uma maior predominância de idosos com limitação na atividade agachar e pegar um lápis no chão $(85,4 \%)$, sem limitação na atividade sentar e levantar $(92,7 \%)$, sem limitação no teste de caminhada $(96,3 \%)$, sem limitação na força de preensão manual $(79,3 \%)$ e sem limitação na atividade de equilíbrio $(81,7 \%)$.

Tabela 3 - Distribuição dos idosos segundo as atividades do desempenho motor. Vitória da Conquista/BA, 2015

\begin{tabular}{lcc}
\hline & $N$ & $\%$ \\
\hline Agachar e pegar o lápis no chão & 140 & 85,4 \\
$\quad$ Com limitação & 24 & 14,6 \\
Sem limitação & & \\
Sentar Levantar & 12 & 7,3 \\
$\quad$ Com limitação & 152 & 92,7 \\
$\quad$ Sem limitação & & \\
Teste de Caminhada & 6 & 3,7 \\
$\quad$ Com limitação & 158 & 96,3 \\
$\quad$ Sem limitação & & \\
Força de Preensão Manual & 34 & 20,7 \\
$\quad$ Com limitação & 130 & 79,3 \\
$\quad$ Sem limitação & & 18,3 \\
Equilíbrio & 30 & 81,7 \\
$\quad$ Com limitação & 134 & 100,0 \\
$\quad$ Sem limitação & 164 & \\
\hline Total & & \\
\hline
\end{tabular}




\section{DISCUSSÃO}

O presente estudo teve como objetivo avaliar o desempenho motor de idosos participantes de um grupo de convivência no munícipio de Vitória da Conquista. O estudo mostrou predomínio de idosos do sexo feminino. Isto se dá em função da maior prevalência de mulheres em comparação ao número de homens idosos e ao fato das mulheres frequentarem mais espaços de grupos sociais comparado aos homens.

A motivação feminina em participar das atividades de lazer é diferente da masculina, uma vez que a maior parte das idosas atuais exerceu um papel restrito ao ambiente doméstico. Ou seja, as muIheres que frequentam grupos de terceira idade em sua maioria não desenvolveram atividades profissionais, ao contrário, foram donas-de-casa, mães e esposas. ${ }^{(9)}$

A importância do lazer, para o idoso, existe em virtude do aumento do tempo livre, pois é por meio deste que os idosos encontram motivação e satisfação em viver mais e com qualidade. Idosos mantêm as mesmas necessidades psicológicas e sociais que possuíam nas outras fases da vida e, por isso, reconhecem e valorizam o lazer. Ter grupo de referência, em que se possam compartilhar alegrias, tristezas, conhecimentos, entre outros, propicia aos idosos suporte emocional e motivação para que tenham objetivos na vida. ${ }^{(10)}$

Melhores condições de vida contribuem para o aumento da expectativa de vida e da longevidade, sendo que as mulheres vivem mais que os homens. Dessa forma, especialmente a longevidade feminina, acarreta um número expressivo de viúvas participando de grupos de convivência. ${ }^{(6)} \mathrm{O}$ perfil sociodemográfico, mostrou que grande parte dos idosos eram viúvos, sendo a sua maioria mulheres.

Em relação a faixa etária houve uma maior frequência de idosos na faixa etária igual ou maior a 68 anos, resultado semelhante ao encontrado em pesquisa realizada em Londrina no qual a média de idade dos idosos foi 69,3 anos para o sexo masculino e 68,4 anos para o sexo feminino. ${ }^{(11)}$
Quanto a renda constatou-se que a amostra estudada tinha renda menor igual a 1 (um) salário mínimo, dados estes semelhantes a estudo realizado em Guarapuava/PR no qual 75,1\% dos idosos possuíam renda de 0 a 1 salário mínimo. ${ }^{(12)}$ Esta realidade limita o acesso a bens de serviços e de consumo, como alimentação, saúde, medicação e moradia adequadas, interferindo de maneira negativa na qualidade de vida dos idosos.

A alta frequência de viúvas pode ser explicada pela elevação progressiva na expectativa de vida da população feminina em detrimento da masculina. Esse fator tanto é relevante que o Ministério da Saúde instituiu, no ano de 2008 , a política de saúde do homem, visando, dentre outros fatores, a diminuir a mortalidade masculina em todas as faixas etárias, a fim de aumentar a expectativa de vida desse grupo, que é sete anos menor que em mulheres. ${ }^{(13)}$

Em relação às condições de saúde verificou-se que houve uma maior frequência de idosos com presença de doenças, com presença de dor e que percebem a sua saúde comparando com pessoas de sua idade como boa. Para Os idosos compõem uma categoria para a qual não se deve generalizar. Qualquer medida de atenção com sua saúde deve levar em conta o indivíduo, o momento e a necessidade de ação específica. ${ }^{(14-15)}$

A capacidade funcional surge, portanto, como um novo paradigma de saúde, particularmente relevante para o idoso. De fato, as doenças crônicas e a capacidade funcional podem ser consideradas determinantes próximos da saúde dos idosos. Esses fatores possuem enorme poder explicativo dos desfechos de saúde. ${ }^{(16)}$

As dores corporais podem interferir na QV da pessoa idosa, influenciando na depressão, na incapacidade física e funcional, na dependência, no afastamento social, em alterações na dinâmica familiar, no desequilíbrio econômico, na desesperança, além de acarretar fadiga, problemas no apetite, alterações de sono, constipação, náuseas, dificuldades de concentração e na execução das atividades da vida diária. ${ }^{(15)}$ 
As atividades em grupo possibilitam aos idosos a socialização e a verbalização de suas experiências de vida tornando um espaço de acolhimento, troca e construção de vínculos. Essas atividades em conjunto influenciam diretamente na melhora da qualidade de vida desses idosos que apresentam doenças relacionadas ao processo de envelhecimento.

A independência funcional está relacionada às habilidades físicas das tarefas simples do cotidiano. $\mathrm{Na}$ avaliação do desempenho motor observou-se uma maior predominância de idosos com limitação na atividade agachar e pegar um lápis no chão, sem limitação na atividade sentar e levantar, sem limitação no teste de caminhada, sem limitação na força de preensão manual e sem limitação na atividade de equilíbrio.

A percepção da própria saúde é forte indicador do estado de saúde dos idosos, porque prediz, de forma consistente, a sobrevida dessa população. Estudos $^{(12-17)}$ demonstraram que a percepção de saúde boa é um dos indicadores mais relatados. No presente estudo, verificou-se uma maior distribuição que consideraram sua saúde como boa, isto pode ser justificado pelo fato dos idosos encontrarem-se ativos e desempenhando atividades de lazer. As atividades físicas desenvolvidas nos centros de convivência funcionam como recurso poderoso contra o envelhecimento do corpo e da mente, eles retomam a auto-estima, melhoram a percepção da saúde e consequentemente a qualidade de vida.

Estudos comprovam que existe uma relação de diminuição da aptidão funcional com o avanço da idade. ${ }^{(2-17)} \mathrm{Em}$ estudo realizado no Pernambuco ${ }^{(18)}$ $51,5 \%$ dos idosos avaliados apresentaram comprometimento funcional, dados estes contraditórios ao do presente estudo do qual os idosos foram classificados sem comprometimento na maioria dos testes de desempenho motor.

$\mathrm{Na}$ avaliação da atividade de agachar e pegar um lápis no chão houve uma maior frequência de idosos classificados com limitação, resultado estudo contraditório ao encontrado em estudo no Paraná com idosos práticas de atividade física no qual $98,0 \%$ dos idosos foram classificados sem limitação. ${ }^{(18)}$
$\mathrm{Na}$ atividade de sentar e levantar verificou-se no presente estudo uma maior frequência de idosos classificados sem limitação, resultado semelhante a estudo realizado com idosos praticantes de atividade física no qual 100,0\% dos idosos foram denominados sem comprometimento. ${ }^{(18)}$

Quanto a atividade de equilíbrio constatou-se que a maioria dos idosos avaliados foram classificados sem comprometimento, dados estes contraditórios a estudo realizado no Maringá no qual $68,0 \%$ dos idosos foram denominados com comprometimento. ${ }^{(18)}$

Em relação a FPM os idosos pesquisados foram em sua maior parte denominados sem comprometimento, resultado este semelhante ao encontrado em estudo realizado em São Paulo, no qual a maioria dos idosos avaliados também foram classificados sem comprometimento, visto que a media foi $35,69 \mathrm{~kg}$ para o sexo masculino e $32,47 \mathrm{~kg}$ para o sexo feminino. ${ }^{(19)}$

No teste de caminhada verificou-se uma maior concentração de idosos denominados sem com prometimento, resultados estes reafirmados por estudo realizado em Londrina no qual a maioria dos idosos avaliados também foram classificados sem comprometimento. ${ }^{(1)}$

O maior comprometimento dos idosos em apenas umas das atividades do desempenho motor pode ser justificado pelo fato que todos os idosos avaliados realizam atividades físicas no grupo de convivência. Frequentar um grupo de convivência possibilita aos idosos conhecerem novas pessoas, construir amizades, viajar acompanhando o grupo, fazer exercícios físicos, divertir-se, dentre outras razões, isso reflete no relacionamento com a família e na disposição física. ${ }^{(20-21)}$

A falta de atividades é um indicador de fragilidade, contribuindo para o declínio da capacidade funcional. Desse modo, as atividades e socialização promovida pela participação no grupo de convivência pode ser uma explicação para a redução limitação funcional dos idosos participantes os grupos de convivência. Tais atividades estimulam o indivíduo a adquirir maior autonomia, melhora sua 
autoestima, qualidade de vida, senso de humor e promove sua inclusão social.

Os dados do presente estudo corroboram estudos anteriores e servem de base para a continuidade de novos focos de investigação, uma vez que se percebe que os idosos participantes de grupos de convivência avaliados apresentaram pouco comprometimento da capacidade motora. Como limitação neste estudo ressalta-se a não divisão dos idosos por sexo e faixa estaria, sendo assim, sugere-se que novos estudos sejam realizados levando-se em consideração estas variáveis.

\section{CONCLUSÃO}

Constatou-se que os idosos avaliados apresentaram limitação no desempenho motor apenas na atividade de atividade agachar e pegar um lápis no chão. De acordo com os resultados obtidos neste estudo, observou-se a importância de os idosos participarem de grupos de convivência a fim de contribuir para uma vida com mais autonomia e independência funcional.

Sendo assim, as atividades proporcionadas pelos grupos, possibilitam aos idosos um estilo de vida mais saudável e, consequentemente, uma meIhoria da qualidade de vida e melhor condição de envelhecimento.

\section{REFERÊNCIAS}

1. Almeida EA, Madeira GD, Arantes PMM, Alencar MA. Comparação da qualidade de vida entre idosos que participam e idosos que não participam de grupos de convivência na cidade de Itabira-MG. Rev. bras. geriatr. gerontol. 2010;13(3):435-444.

2. Instituto Brasileiro de Geografia e Estatística. Perfil dos idosos responsáveis pelos domicílios no Brasil [Internet]. $2 \mathrm{O} 10$ [acesso em O2 fev. 2014]. Disponível em: http://www.ibge.gov.br

3. Carmo LV, Drummond LP, Arantes PM. Avaliação do nível de fragilidade em idosos participantes de um grupo de convivência. Fisioter. pesqui. 2011;18(1):17-22.

4. Soares LDA, Campos FAAS, Araújo MGRA, Falcão APST, Lima BRDA, Siqueira DF, Fittipaldi EOS, Arruda SGB, Faro ZP. Análise do Desempenho Motor associado ao Estado Nutricional de Idosos cadastrados no Programa Saúde da Família, no município de Vitória de Santo Antão-PE. Ciênc. saúde coletiva. 2O12;17(5):1297-1304.

5. Pinheiro PA, Passos TODO, Coqueiro RS, Fernandes MH, Barbosa RA. Desempenho motor de idosos do Nordeste brasileiro: diferenças entre idade e sexo. Rev. Esc. Enferm. USP. 2O13; 47(1):128-36.

6. Benedetti TRB, Mazo GZ, Borges LJ. Condições de saúde e nível de atividade física em idosos participantes e não participantes de grupos de convivência de Florianópolis. Ciênc. saúde coletiva. 2012;17(8):2087-2093.

7. Pfeiffer, E. A short portable mental status questionnaire for the assessment of organic brain deficit in elderly patients. J. am. geriatr. soc.1975; 23(10):433-41.

8. Neumann B, Conde SR, Lemos JRN, Moreira TR. Associação entre o estado nutricional e a prevalência de doenças crônicas não transmissíveis em idosos residentes no município de Roca Sales-RS. RBCEH, 2O14; 166-177.

9. Leite MT, Hilderbrandt LM, Kirchner RM, Winck MT, Silva LAA, Franco GP: Estado cognitivo e condições de saúde de idosos que participam de grupos de convivência. Rev. gaúch. enferm. 2012; 33(4):64-71.

10. Andrade NA, Nascimento MMP, Oliveira MMD, Queiroga RM, Fonseca FLA, Lacerda SNB, Adami F. Percepção de idosos sobre grupo de convivência: estudo na cidade de CajazeirasPB. Rev. bras. geriatr. gerontol. 2014;17(1):39-48.

11. Travensolo CF, Goessler KF, Marcos Doederlein PolitoMD. Correlação entre o teste de caminhada de seis minutos e o teste do degrau em idosos. Rev. bras. geriatr. gerontol. 2013;16(2):375-383.

12. Pilger $\mathrm{C}$, Menon MH, Mathias TAF. Características sociodemográficas e de saúde 
de idosos: contribuições para os serviços de saúde. Rev. latinoam. enferm. 2011;19(5).

13. Silva HO, Carvalho MJAD, Lima FELL, Rodrigues LV. Perfil epidemiológico de idosos frequentadores de grupos de convivência no município de lguatu, Ceará. Rev. bras. geriatr. gerontol. 2011;14(1):123-133.

14. Gutierrez BAO, Auricchio AM, Medina NVJ. Mensuração da qualidade de vida de idosos em centros de convivência. J health. sci. inst. 2011;29(3):186-90.

15. Krug RR, Lopes MA, Mazo GZ, Marchesan M. Pain impairs the practice of regular physical activities in the perception of longevous womenA dor dificulta a prática de atividade física regular na percepção de idosas longevas. Rev. dor. 2013;14(3):192-5.

16. Alves LC, Leite IC, Machado CJ. Perfis de saúde dos idosos no Brasil: análise da Pesquisa Nacional por Amostra de Domicílios de 2003 utilizando o método Grade of Membership. Cad. saúde pública. 2008;24(3):535-546.
17. Soares LDA, Campos FACS, Araújo MGR, Falcão APST, Lima BRDA, Siqueira DF et al. Análise do desempenho motor associado ao estado nutricional de idosos cadastrados no Programa Saúde da Família, no município de Vitória de Santo Antão-PE. Ciênc. saúde coletiva. 2012;17(5):1297-1304.

18. Souza FF, Bertoline SMMG. Desempenho motor de idosos praticantes de atividades físicas em academias da terceira idade da cidade de Maringá, Paraná. Anais Eletrônico. VI Mostra Interna de Trabalhos de Iniciação Científica.

19. Fabíola Giannattasio Martin FG, Nebuloni CC, Najas MS. Correlação entre estado nutricional e força de preensão palmar em idosos. Rev. bras. geriatr. gerontol. 2012;15(3):493-504

2O. Soares LDA et al. Análise do desempenho motor associado ao estado nutricional de idosos cadastrados no Programa Saúde da Família, no município de Vitória de Santo Antão-PE. Ciênc. saúde coletiva. 2012;17(5).

21. Rizzolli D; Surdi A C: Percepção dos idosos sobre grupos de terceira idade. Rev. bras. geriatr. gerontol. 2010;13(2):225-233. 